\title{
Alguns fungos conidiais aquáticos-facultativos do bioma Caatinga
}

\author{
Davi Augusto Carneiro Almeida ${ }^{1}$, Flavia Rodrigues Barbosa ${ }^{2}$ e Luís Fernando Pascholati Gusmão ${ }^{1,3}$
}

Recebido em 11/09/2011. Aceito em 20/07/2012

\begin{abstract}
RESUMO
(Alguns fungos conidiais aquáticos-facultativos do bioma Caatinga). Durante o estudo de fungos conidiais associados a substratos vegetais submersos na Serra da Fumaça e na Serra da Jibóia, estado da Bahia, 17 espécies foram identificadas, sendo cinco novos registros: Actinocladium verruculosum para o continente americano, Xylomyces aquaticus para o Neotrópico, Actinocladium longiramosum e Dischloridium inaequiseptatum para a América do Sul e Cacumisporium pleuroconidiophorum para o Brasil. Descrições, ilustrações, distribuição geográfica e comentários são apresentados para as espécies acima, bem como uma lista com as demais espécies encontradas.
\end{abstract}

Palavras-chave: biodiversidade, hifomicetos, substrato submerso, taxonomia

\begin{abstract}
(Some facultative-aquatic conidial fungi from the Caatinga Biome). During study of conidial fungi on submerged plant debris in Serra da Fumaça and Serra da Jibóia, Bahia, 17 species were identified. These species included five new records: Actinocladium verruculosum for the American continent, Xylomyces aquaticus for the Neotropics, Actinocladium longiramosum and Dischloridium inaequiseptatum for South America and Cacumisporium pleuroconidiophorum for Brazil. Descriptions, illustrations, geographic distributions and comments are provided for the aforementioned species and a list of all of the species is included.
\end{abstract}

Key words: biodiversity, hyphomycetes, submerged substrate, taxonomy

\section{Introdução}

Os fungos conidiais aquáticos constituem um grupo polifilético (Belliveau \& Bärlocher 2005) que é encontrado nas folhas e demais substratos vegetais submersos em decomposição nos rios de todo o mundo (Medeiros et al. 2009). Os fungos aquáticos são divididos basicamente em quatro grupos de acordo com sua morfologia e estilo de vida: fungos ingoldianos, fungos aeroaquáticos (Alexopoulos 1996), fungos aquático-terrestres e fungos aquático-facultativos (Goh \& Hyde 1996).

Os fungos ingoldianos ocorrem frequentemente em ambientes lóticos com boa aeração e estão entre os poucos grupos de fungos cujas espécies podem, em muitos casos, ser identificadas baseando-se apenas na observação dos conídios (Descals 2005). Seus conídios apresentam adaptações morfológicas à dispersão nesse ambiente, sendo tipicamente ramificados ou sigmóides e acumulam-se facilmente em espumas que se formam na superfície da água (Ingold 1966). Algumas espécies também podem ser encontradas, menos frequentemente, em ambientes lênticos ou não aquáticos (Descals \& Moralejo 2001). No Brasil, entre as poucas espécies registradas até o momento é possível citar: Alatospora acuminata Ingold, Anguillospora crassa Ingold, A. longissima (Sacc. \& P. Syd.) Ingold, Articulospora tetracladia Ingold, Clavariopsis aquatica De Wild., Flagellospora crassa Alasoadura, F. curvula Ingold e F. penicillioides Ingold (Schoenlein-Crusius \& Grandi 2003).

Os fungos aeroaquáticos são geralmente encontrados em ambientes lênticos como lagos estagnados e riachos com fluxo lento, crescendo sobre folhas ou madeiras submersas, mas também podem ser encontrados em todos os tipos de habitats aquáticos e/ou terrestres (Shearer et al. 2004). O gênero aeroaquático Helicosporium Nees (Webster \& Descals 1981; Dix \& Webster

\footnotetext{
${ }^{1}$ Universidade Estadual de Feira de Santana, Laboratório de Micologia, Feira de Santana, BA, Brasil

${ }^{2}$ Universidade Estadual do Sudoeste da Bahia, Departamento de Ciências Biológicas, Jequié, BA, Brasil

${ }^{3}$ Autor para correspondência: lgusmao@uefs.br
} 
1995; Tsui \& Berbee 2006), por exemplo, já foi isolado de madeiras e folhas em ambiente terrestre no bioma Caatinga, por Cruz \& Gusmão (2009a, b) e Cruz et al. (2009). Esse grupo de fungos aeroaquáticos geralmente produz conídios com formas helicoidais (helicosporos), esporulando apenas quando o substrato é exposto ao ar (Webster \& Descals 1981; Ando \& Tubaki 1984; Alexopoulos 1996).

Os fungos aquático-terrestres incluem várias espécies encontradas na água da chuva ou orvalho acumulada na superfície de folhas (Ando 1992). Esse grupo produz estaurosporos morfologicamente similares aos conídios produzidos pelos fungos ingoldianos, mas diferenciam-se principalmente pela ausência de conidióforos macronemáticos (Ando 1992; Goh \& Hyde 1996; Descals \& Moralejo 2001). Exemplos deste grupo são os gêneros: Alatosessilispora K. Ando \& Tubaki, Arborispora K. Ando, Curucispora Matsush., Microstella K. Ando \& Tubaki, Ordus K. Ando \& Tubaki, Tricladiella K. Ando \& Tubaki e Trifurcospora K. Ando \& Tubaki (Ando \& Tubaki 1984; Ando 1992). No entanto, nenhum desses gêneros foi relatado para o Brasil.

Os fungos aquático-facultativos são representados por hifomicetos dematiáceos, crescendo como sapróbios em substratos vegetais submersos ou terrestres (principalmente lignificados) e esporulando sob condições terrestres ou, em alguns casos, também submersas. As estruturas reprodutivas (conídios e conidióforos) apresentam parede relativamente espessa e os conídios são capazes de dispersão tanto na água quanto no ar (Webster \& Descals 1981; Goh \& Hyde 1996; Descals \& Moralejo 2001; Révay \& Gönczöl 2007). Também são chamados de hifomicetos lignícolas ou hifomicetos aquáticos-submersos (Ingold 1975; Goh \& Hyde 1996). Segundo Goh \& Hyde (1996), gêneros representativos desse grupo são Bactrodesmium Cooke, Brachydesmiella G. Arnaud ex S. Hughes, Brachysporiella Bat., Camposporidium Nawawi \& Kuthub., Canalisporium Nawawi \& Kuthub., Cryptophiale Piroz., Cryptophialoidea Kuthub. \& Nawawi, Dactylaria Sacc., Dendryphiosphaera Lunghini \& Rambelli, Dictyochaeta Speg., Exserticlava S. Hughes, Kionochaeta P.M. Kirk \& B. Sutton, Monotosporella S. Hughes, Nawawia Marvanová, Phaeoisaria Höhn., Spadicoides S. Hughes, Sporidesmiella P.M. Kirk, Sporidesmium Link, Sporoschisma Berk. \& Broom, Sporoschismopsis Hol.-Jech. \& Hennebert, Trichocladium Harz e Xylomyces Goos, R.D. Brooks \& Lamore. Alguns desses gêneros são frequentemente encontrados no Brasil.

Os restos vegetais alóctones (folhas, frutos, galhos e troncos) são uma importante fonte de energia em ecossistemas dulcícolas (Wallace et al. 1997, Bärlocher 2009, Cole et al.2010), principalmente aqueles onde a sombra formada pela mata ciliar limita a produção primária pela fotossíntese (Webster \& Meyer 1997, Schindler \& Gessner 2009). Desse modo, eles são de fundamental importância para a manutenção da cadeia trófica nesses ambientes (Benke et al. 1988). Os fungos aquáticos têm papel crucial na decomposição desses substratos, transformando-os em uma fonte alimentar mais palatável e de maior qualidade nutricional para invertebrados detritívoros (Boling et al. 1975, Benke et al. 1988). De fato, alguns trabalhos demonstraram a preferência de invertebrados detritívoros por substratos vegetais colonizados por fungos (Bärlocher \& Kendrick 1975; Chung \& Suberkropp 2009). Essa função ecológica pode ser atribuída à habilidade dos fungos em produzir enzimas capazes de degradar polímeros estruturais como a celulose, lignina, hemicelulose e pectina (Torzilli 1982, Suberkrop et al. 1983, Benner et al. 1984). Apesar da sua importância para os ecossistemas dulcícolas, o conhecimento sobre a diversidade dos fungos conidiais aquáticos das regiões tropicais permanece precário, uma vez que a maioria dos estudos tem sido conduzida em regiões temperadas (Chan et al. 2000; Mathuria \& Chauvet 2002; Tsui et al. 2003).

Em uma compilação de dados da literatura, Schoenlein-Crusius \& Grandi (2003) apresentaram um checklist com 90 espécies de hifomicetos aquáticos registradas na América do Sul, incluindo Brasil, Chile, Equador, Peru e Venezuela. Essas autoras enfatizaram a forte necessidade do aprimoramento dos conhecimentos sobre a diversidade de hifomicetos aquáticos nesse continente, uma vez que artigos publicados ainda são esporádicos e dispersos. No Brasil, a maioria dos estudos realizados estão concentrados no estado de São Paulo (Schoenlein-Crusius et al. 2009). Para o bioma Caatinga, embora uma série de estudos sobre a diversidade de fungos conidiais tenham sido conduzidos na primeira década do século XXI, nenhum deles tratou sobre os fungos conidiais ocorrendo em ambientes aquáticos (Gusmão \& Grandi 2001; Barbosa \& Gusmão 2005; Gusmão et al. 2005; Gusmão \& Barbosa 2005; Castañeda-Ruiz et al. 2006; Barbosa et al. 2007; Cruz et al. 2007a, b, c; Marques et al. 2007; Barbosa et al. 2008; Cruz et al. 2008a, b; Gusmão et al. 2008; Marques et al. 2008; Barbosa et al. 2009a, b; Cruz \& Gusmão 2009a, b; Leão-Ferreira \& Gusmão 2010). Dessa forma, o presente trabalho tem o objetivo de contribuir para ampliar o conhecimento sobre a distribuição geográfica e a diversidade dos fungos conidiais aquático-facultativos do bioma Caatinga, sendo pioneiro no que tange o habitat submerso para o bioma.

\section{Materiais e métodos}

\section{Área de Estudo}

A Serra da Fumaça e a Serra da Jibóia estão localizadas no bioma Caatinga e consequentemente na região semi-árida brasileira. A Serra da Fumaça ( $\left(0^{\circ} 39^{\prime}\right.$ S e 402으) está situada no município de Pindobaçu, estado da Bahia, e integra uma cadeia de serras denominada de Serra de Jacobina, a qual se estende por cerca de $200 \mathrm{Km}$ no sentido norte-sul na porção norte da Bahia, com 15-25 Km de largura. Sua altitude pode alcançar até $1.300 \mathrm{~m}$ (Mascarenhas et al. 1998; Milesi et al. 2002). A Serra da Jibóia está situada na região do Recôncavo Sul, porção leste da Bahia, e se distribui ao 
longo do território de seis municípios, apresentando uma área de aproximadamente 22.000 ha e altitude entre 750 e $840 \mathrm{~m}$ (Neves 2005). Os estudos foram desenvolvidos ao longo de $150 \mathrm{~m}$ de um riacho localizado no Monte da Pioneira ( $12^{\circ} 51^{\prime}$ S e $\left.39^{\circ} 28^{\prime} \mathrm{O}\right)$, município de Santa Teresinha.

\section{Método de amostragem}

Amostras de materiais vegetais submersos em decomposição (folhas, galhos e cascas) foram coletadas em 2007 e 2009, acondicionadas em sacos plásticos e transportadas ao laboratório de micologia de UEFS. As amostras foram lavadas em água corrente por 30 min e em seguida colocadas em placas de Petri com papel filtro umedecido, constituindo-se em câmaras-úmidas. Essas foram então colocadas em uma caixa de isopor ( $170 \mathrm{~L}$ de capacidade) revestida internamente com papel toalha umedecido e com o fundo recoberto por $500 \mathrm{~mL}$ de água, acrescido de $2 \mathrm{~mL}$ de glicerol para manutenção da umidade (Castañeda-Ruiz 2005). Durante um período de 30 dias, as amostras foram examinadas diariamente em busca de estruturas reprodutivas dos fungos conidiais. Lâminas permanentes foram confeccionadas com resina PVL (álcool polivinílico + ácido lático + fenol) e depositadas no Herbário da Universidade Estadual de Feira de Santana (HUEFS) junto com as amostras vegetais secas. Fotomicrografias foram obtidas utilizando uma câmera digital Canon G5 acoplada ao microscópio óptico Axioscop 40 Carl Zeiss. As ilustrações foram confeccionadas baseadas no método descrito por Barber \& Keane (2007).

\section{Resultados e discussão}

Foram identificadas 17 espécies de fungos conidiais associadas à decomposição de substratos vegetais em ambiente aquático. Destas, cinco constituem novos registros distribuídos entre o continente americano, Neotrópico, América do Sul e Brasil. As demais espécies já foram previamente registradas no bioma Caatinga sobre substratos vegetais coletados em ambiente terrestre (Gusmão 2003; Barbosa et al. 2007; Cruz et al. 2008a; Gusmão et al. 2008; Barbosa et al. 2009a; Cruz \& Gusmão 2009a, b), contudo estão sendo citadas aqui pela primeira vez em ambiente aquático nesse bioma.

Actinocladium longiramosum (R.F. Castañeda) R.F. Castañeda, Mycotaxon 60: 278. 1996.

Bas.: Ceratosporella longiramosa R.F. Castañeda, Fungi Cubenses III (La Habana): 2. 1988.

Fig. 1a-d e 2a-c

Conidióforos macronemáticos, mononemáticos, retos, simples, eretos, 1-2 septados, lisos, castanhos na base, subhialianos no ápice, $15-22,5 \times 4,5-6 \mu \mathrm{m}$. Células conidiogênicas monoblásticas, terminais, integradas, lisas, cilíndricas, subhialinas. Conídios solitários, esquizolíticos, secos, estaurosporos, constituídos por uma célula basal e 2-3 ramificações divergentes, subhialinos; ramificações lisas, retas, 5-12-pseudoseptadas, subuladas, subhialinas, 87-294 $\times 4-5 \mu \mathrm{m}$; células basais cônico-cilíndricas, truncadas na base, lisas, 4,5-6 $64-6,5 \mu \mathrm{m}$.

Wu \& Zhuang (2005) consideraram cinco espécies no gênero: A. amazonicum Matsush., A. atrosporum G.C. Zhao \& N. Li, A. longiramosum, A. rhodosporum Ehrenb. e A. verruculosum W.P. Wu. Destas, A. longiramosum é a única espécie que apresenta conídios pseudoseptados (Ellis 1971; Castañeda-Ruiz 1988; Matsushima 1993; Wu \& Zhuang 2005). Actinocladium Ehrenb. é semelhante à Ceratosporella Höhn., mas difere pela morfologia dos conídios. As espécies de Actinocladium produzem estaurósporos, enquanto as de Ceratosporella produzem conídios queiróides. Com base nessa diferença, Castañeda-Ruiz et al. (1996) transferiu Ceratosporella longiramosa R.F. Castañeda para o gênero Actinocladium. No material brasileiro, as ramificações dos conídios atingiram comprimentos maiores do que o relatado nas descrições consultadas (Ellis 1971; Castañeda-Ruiz 1988; Matsushima 1993; Wu \& Zhuang 2005). Células conidiogênicas não apresentaram extensões percurrentes provavelmente devido à sua maturidade. Esse é o primeiro registro da espécie para a América do Sul e em substrato lignícola submerso.

Distribuição geográfica conhecida: Cuba (Castañeda-Ruiz 1988).

Material examinado: BRASIL. Bahia: Pindobaçu, Serra da Fumaça, sobre casca submersa em decomposição, 19.XI.2008, D.A.C. Almeida s.n. (HUEFS154966).

Actinocladium verruculosum W.P. Wu, Fung. Divers. Res. Ser. 15: 228. 2005.

Fig. 1e-g e $2 \mathrm{~d}$

Conidióforos macronemáticos, mononemáticos, retos ou levemente flexuosos, simples, eretos, 3-6 septados, lisos, castanhos, $30-85 \times 5-12,5 \mu \mathrm{m}$; ápice 5-7,5 $\mu \mathrm{m}$ de largura. Células conidiogênicas monoblásticas, terminais, integradas, $0-2$ extensões percurrentes, lisas, cilíndricas a lageniformes, truncadas no ápice, castanhas. Conídios solitários, esquizolíticos, estaurósporos, secos, constituídos por um corpo central com 1-2 células, com 3-7 ramificações; ramificações retas, divergentes, verrucosas, 8-9 septadas, cilíndricas a subuladas, ápices arredondados a truncados, castanhas na base, castanho-claras no ápice, 27,5-95 $\times$ 7,5-10 $\mu \mathrm{m}$; corpos centrais cilíndricos a clavados, lisos, castanhos, $10-13 \times 7-10 \mu \mathrm{m}$; célula basal obcônica e truncada, 5-10 $\times 4-5 \mu \mathrm{m}$.

Actinocladium verruculosum foi proposto por $\mathrm{Wu} \&$ Zhuang (2005) para um espécime isolado sobre folhas em decomposição na China. É facilmente distinguida das demais espécies do gênero pelos conídios conspicuamente verrucosos. Actinocladium rhodosporum Ehrenb. é similar a A. verruculosum, mas difere pelos conídios lisos, além do menor número de ramificações (3-4) (Ellis 1971). Yurchenko (2001) coletou um espécime com estaurósporos 
verrucosos na Bielorrússia, o qual ele identificou como $A$. rhodosporum, no entanto, baseado na descrição e ilustração apresentada por este autor, concluímos que este espécime é $A$. verruculosum.

As características do material brasileiro estão de acordo com as descritas por Wu \& Zhuang (2005), com exceção dos conidióforos que alcançaram comprimentos e larguras maiores. Este é o primeiro registro da espécie para o continente Americano e sobre substrato lignícola submerso.
Distribuição geográfica conhecida: Bielorrusia (como A. rhodosporum, Yurchenko, 2001), China (Wu \& Zhuang 2005).

Material examinado: BRASIL. Bahia: Santa Teresinha, Serra da Jibóia, sobre galho submerso em decomposição, 28.VIII.2007, F.R. Barbosa s.n. (HUEFS155257). Pindobaçu, Serra da Fumaça, sobre casca terrestre em decomposição, 20.XI. 2008, D.A.C. Almeida s.n. (HUEFS154967).
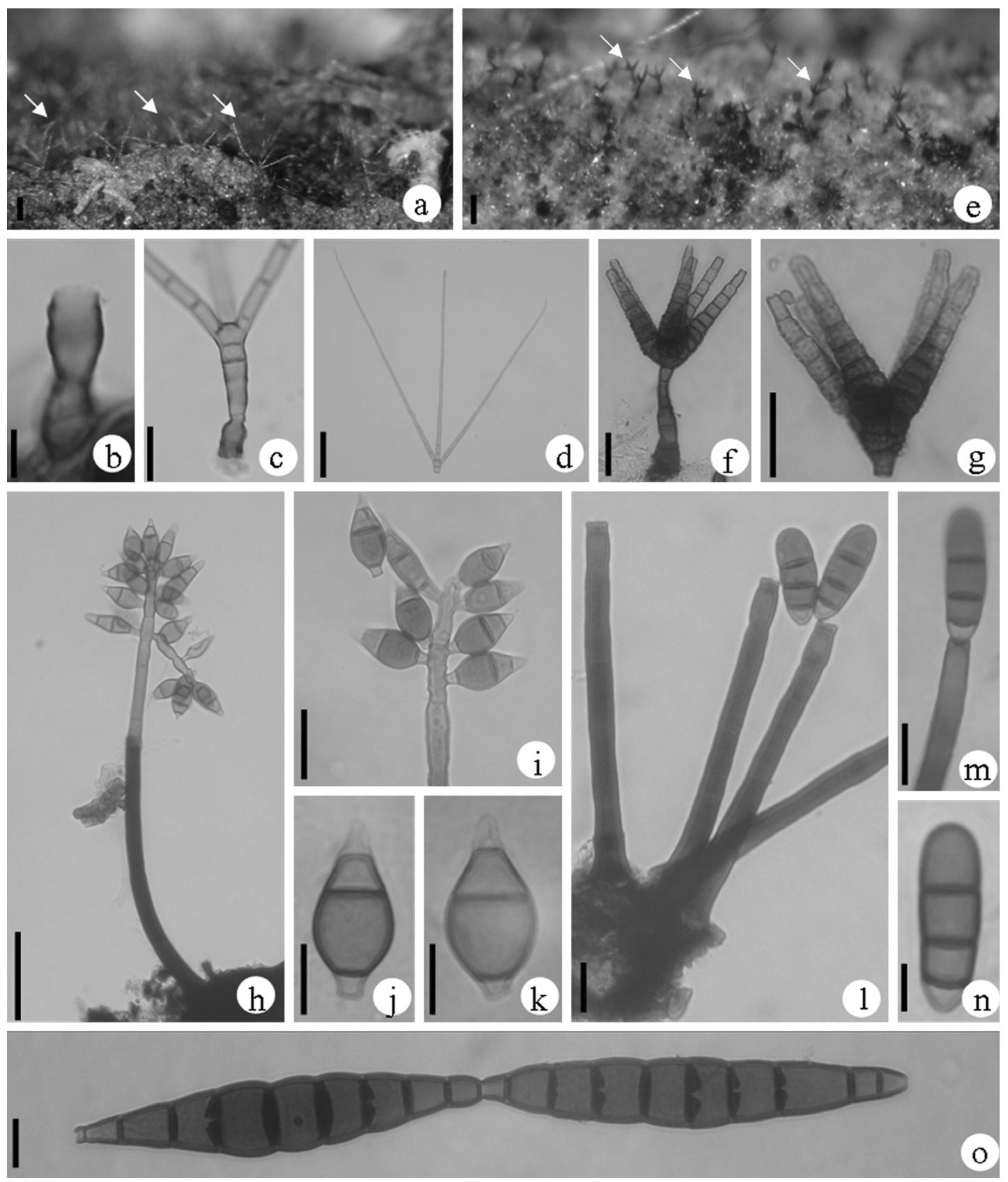

Figura 1. a-d. Actinocladium longiramosum (R.F. Castañeda) R.F. Castañeda - a. conídios sobre substrato natural (setas); b. conidióforo c. conidióforo com conídio; d. conídio. e-g. Actinocladium verruculosum W.P. Wu - e. conídios sobre substrato natural (setas); f. aspecto geral; g. conídio. h-k. Cacumisporium pleuroconidiophorum (Davydkina \& Melnik) R.F. Castañeda, Heredia \& Iturr. - h. aspecto geral; i. ápice do conidióforo com conídios; j-k. conídios. l-n. Dischloridium inaequiseptatum (Matsush.) Hol.-Jech. - l. aspecto geral; m. ápice do conidióforo com conídio; n. conídio. o. Xylomyces aquaticus (Dudka) K. D. Hyde \& Goh - o. clamidósporos. Barras: 50 mm (a, d-e, h); 20 $\mu \mathrm{m}(\mathrm{f}-\mathrm{g}, \mathrm{i}) ; 10 \mu \mathrm{m}(\mathrm{c}, \mathrm{j}-\mathrm{k}, \mathrm{l}-\mathrm{m}, \mathrm{o}) ; 5 \mu \mathrm{m}(\mathrm{b}, \mathrm{n})$. 
Cacumisporium pleuroconidiophorum (Davydkina \& Melnik) R.F. Castañeda, Heredia \& Iturr., Mycotaxon 100: 332. 2007. Bas.: Pyriculariopsis pleuroconidiophora Davydkina \& Melnik, Mikol. Fitopatol. 23(2): 112. 1989. Sin.: Cacumisporium curvularioides R.F. Castañeda \& W.B. Kendr., Univ. Waterloo Biol. Ser. 35: 16. 1991.

Fig. 1h-k e 2e-f

Conidióforos macronemáticos, mononemáticos, retos ou flexuosos, simples ou ramificados, eretos, 7-19 septados, lisos, castanho-escuros na base, castanho-claros a subhialinos no ápice, $212-700 \times 7,5-10 \mu \mathrm{m}$. Células conidiogênicas poliblásticas, terminais, integradas, simpodiais, cilíndricas, lisas, castanha-claras a subhialinas. Conídios solitários, esquizolíticos, secos, obturbinados, lisos, simples, 3-septados; células basais cônico-cilíndricas, truncadas, retas ou ocasionalmente inclinadas, subhialinas; células centrais castanhas à castanho-claras; células apicais cônicas, com ápice arredondado, subhialinas; 20-30 × 7,5-15 $\mu \mathrm{m}$;

O gênero Cacumisporium Preuss congrega seis espécies: C. capitulatum (Corda) S. Hughes; C. pleuroconidiophorum; C. rugosum K.M. Tsui, Goh, K.D. Hyde \& Hodgkiss; C. sigmoideum Mercado \& R.F. Castañeda, C. spooneri P.M. Kirk e C. tropicale R.F. Castañeda, Gusmão \& Stchigel (Hughes 1958; Mercado-Sierra \& Castañeda-Ruiz 1987; Kirk 1992; Tsui et al. 2001; Castañeda-Ruiz et al. 2007a, b). Cacumisporium sigmoideum é a espécie mais semelhante à C. pleuroconidiophorum, diferindo por apresentar conídios maiores (26-41 $\mu \mathrm{m}$ compr.) com células centrais concolores e célula basal curvada. $\mathrm{O}$ material brasileiro apresenta-se de acordo com a descrição de Castañeda-Ruiz \& Kendrick (1991). Esta espécie está sendo referida pela primeira vez para o Brasil.

Distribuição geográfica conhecida: Argentina (como $C$. curvularioides, Godeas \& Arambarri 2007), Antiga União das Repúblicas Socialistas Soviéticas (como Pyriculariopsis pleuroconidiophora, Farr et al. 2009), Cuba (como C. curvularioides, Castañeda-Ruiz \& Kendrick 1991), México, Venezuela (Castañeda-Ruiz et al. 2007a).

Material examinado: BRASIL. Bahia: Santa Teresinha, Serra da Jibóia, sobre casca submersa em decomposição, 06.IX.2007, F.R. Barbosa s.n. (HUEFS155263); sobre galho submerso em decomposição, 21.XII.2007, F.R. Barbosa s.n. (HUEFS155258). Pindobaçu, Serra da Fumaça, sobre casca terrestre em decomposição, 05.I.2009, D.A.C. Almeida s.n. (HUEFS154968).

Dischloridium inaequiseptatum (Matsush.) Hol.-Jech., Česká Mykol. 41(2): 111. 1987.

Bas.: Endophragmia inaequiseptata Matsush., Icon. micro-

fung. Matsush. lect. (Kobe): 69. 1975.

Fig. 1l-n e 2g-h

Conidióforos macronemáticos, mononemáticos, retos, simples, eretos, septados, lisos, castanho-escuros na base, castanhos no ápice, 70-130 × 4-6 $\mu \mathrm{m}$. Células conidiogêni- cas monoblásticas, fialídicas, terminais, integradas, com (01) extensão percurrente, cilíndricas, com uma leve constrição no ápice, castanhas. Conídios solitários, esquizolíticos, 3-septados, cilíndrico-elipsóides, lisos, retos ou levemente curvos, ápice arredondado, célula basal cônico-truncada e castanho-clara, demais células castanhas, 15-22,5 × 5-7 $\mu \mathrm{m}$.

O gênero Dischloridium B. Sutton é constituído por 15 espécies, apresentando ampla distribuição geográfica sobre substratos folícolas e lignícolas (Kirk 1985; Seifert \& Gams 1985; Mena-Portales \& Mercado-Sierra 1987; Bhat \& Kendrick 1993; Sivanesan \& Alcorn 2002; Schubert \& Braun 2005). Dischloridium ychaffrei (Bhat \& B. Sutton) Hol.-Jech. e D. triseptatum Hol.-Jech. são similares à $D$. inaequiseptatum pela produção de conídios 3-septados. Contudo, $D$. inaequiseptatum difere de ambas as espécies pelos conídios assimetricamente septados, mais estreitos, castanhos e com célula basal subhialina. As características do material examinado concordam com a descrição de Matsushima (1975). Esta espécie esta sendo referida pela primeira vez para a América do Sul.

Distribuição geográfica conhecida: Cuba (Mercado-Sierra et al. 1997), China (como E. inaequiseptata, Farr et al. 2009), Japão (como E. inaequiseptata, Matsushima 1975).

Material examinado: BRASIL. Bahia: Pindobaçu, Serra da Fumaça, sobre folha submersa em decomposição de dicotiledônea não identificada, 13.I.2009, D.A.C. Almeida s.n. (HUEFS154971). Santa Teresinha, Serra da Jibóia, sobre casca submersa em decomposição, 19.XI.2009, F.R Barbosa s.n. (HUEFS155259).

Xylomyces aquaticus (Dudka) K. D. Hyde \& Goh, Mycol. Res. 103(12): 1573. 1999.

Bas.: Camposporium aquaticum Dudka, Ukr. bot. Zh. 23:

91. 1966. Sin.: Vargamyces aquaticus (Dudka) Tóth, Acta Mus. Silesiae, Ser. A 25(3-4):403. 1979.

Fig. 1 o e $2 \mathrm{i}$

Conidióforos, célula conidiogênicas e conídios ausentes. Clamidósporos em cadeia, 9-13 septados, fusiformes, simples, retos, secos, lisos, constrictos nos septos, castanhos a castanho-claros, 82,5-125 x 10-15 $\mu \mathrm{m}$.

O gênero Xylomyces Goos, Brooks \& Lamore possui oito espécies, sendo X. chlamydosporis a espécie-tipo. Caracteriza-se pela ausência de conídios, conidióforos e células conidiogênicas e pela produção de clamidósporos multiseptados, fusiformes e castanhos. Devido à ausência de conídios, Xylomyces é considerado membro dos agonomicetos (Goos et al. 1977). Com exceção de X. foliicola W.B. Kendr. \& R.F. Castañeda, que foi encontrada sobre folha em ambiente terrestre (Castañeda-Ruiz \& Kendrick 1990), todas as outras sete espécies que constituem o gênero foram isoladas de madeira submersa em corpos d'água (Goos et al. 1977; Goh et al. 1997; Kohlmeyer \& Volkmann-Kohlmeyer 1998; Hyde \& Goh 1999). Xylomyces aquaticus tem sido isolada sobre madeira e folhas submersas em decomposição (Hyde \& Goh 1999; Gönczöl et al. 1990). A transferência 


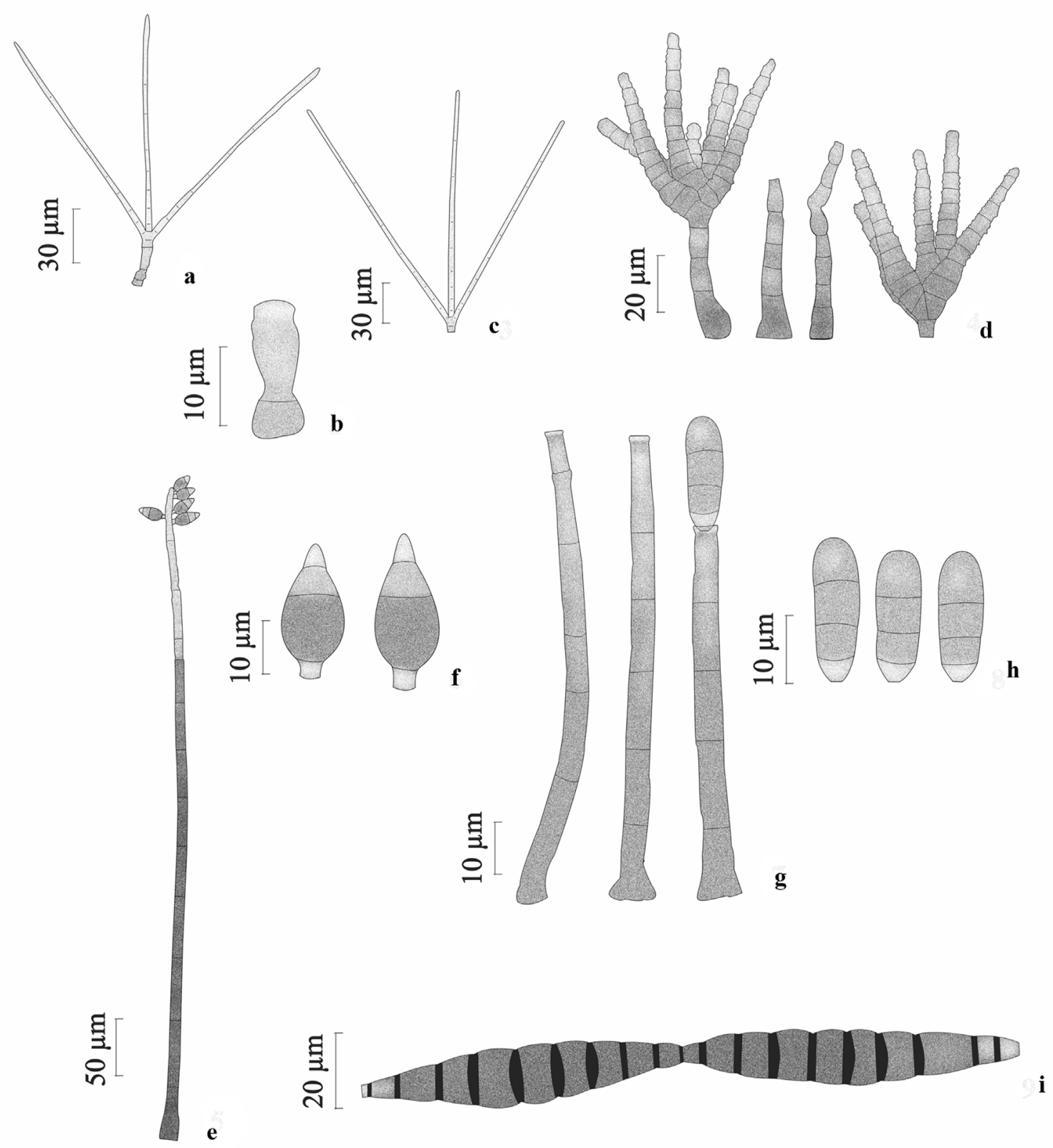

Figura 2. a-c. Actinocladium longiramosum (R.F. Castañeda) R.F. Castañeda. d. Actinocladium verruculosum W.P. Wu. e-f Cacumisporium pleuroconidiophorum (Davydkina \& Melnik) R.F. Castañeda, Heredia \& Iturr. g-h Dischloridium inaequiseptatum (Matsush.) Hol.-Jech. i. Xylomyces aquaticus (Dudka) K. D. Hyde \& Goh.

dessa espécie para o gênero Xylomyces por Hyde \& Goh (1999) implica que os propágulos, anteriormente interpretados como conídios, são clamidósporos em cadeia (Cooper 2005). O material brasileiro foi isolado sobre folhas submersas na Serra da Fumaça e apresentou clamidósporos mais estreitos do que os relatados por outros autores (Gönczöl et al. 1990; Cooper 2005). Os clamidósporos de X. aquaticus são morfologicamente similares aos de X. foliicola, mas diferem pela ocorrência de leves constrições nos septos, maior largura e hábitat aquático. Esta espécie esta sendo citada pela primeira vez para o neotrópico.
Distribuição geográfica conhecida: Bielorrússia (como V. aquaticus, Gulis 2001), Estados Unidos da América (como V. aquaticus, Shearer \& Lane 1983), Hungria (como V. aquaticus, Gönczöl \& Révay 2003), Inglaterra (Hyde \& Goh 1999), Irã (como V. aquaticus, Zare-Maivan \& Ghaderian 1993), Nova Zelândia (Cooper 2005), Polônia (Farr et al. 2009), Tailândia (Kodsueb et al. 2008).

Material examinado: BRASIL. Bahia: Pindobaçú, Serra da Fumaça, sobre folha submersa de dicotiledônea não identificada, 10.XI.2008, D.A.C. Almeida s.n. (UEFS154981); 21.XI.2008, D.A.C. Almeida s.n. (UEFS154982). 
Outras espécies de fungos conidiais encontradas em substrato vegetal submerso nas áreas estudadas:

Atrosetaphiale flagelliformis Matsush., Matsush. Mycol. Mem. 8: 14. 1995.

Material examinado: (HUEFS 154988).

Beltrania rhombica Penz., Michelia 2(8): 474. 1882.

Material examinado: (HUEFS 154993).

Beltraniella portoricensis (F. Stevens) Piroz. \& S.D. Patil,

Can. J. Bot. 48(3): 575. 1970.

Material examinado: (HUEFS 155006).

Chaetopsina fulva Rambelli, Diagn. IV 3: 5. 1956.

Material examinado: (HUEFS 155013).

Chalara alabamensis Morgan-Jones \& E.G. Ingram, Mycotaxon 4(2): 489. 1976.

Material examinado: (HUEFS 155017).

Exserticlava vasiformis (Matsush.) S. Hughes, N.Z. Jl Bot. 16(3): 332. 1978.

Material examinado: (HUEFS 155040).

Kionochaeta pughii Kuthub. \& Nawawi, Trans. Br. mycol.

Soc. 90(3): 437. 1988.

Material examinado: (HUEFS 155044).

Kionochaeta ramifera (Matsush.) P.M. Kirk \& B. Sutton,

Trans. Br. mycol. Soc. 85(4): 715. 1985.

Material examinado: (HUEFS 155047).

Paliphora intermedia Alcorn, Mycotaxon 59: 145. 1996.

Material examinado: (HUEFS 155053).

Paraceratocladium silvestre R.F. Castañeda, Fungi Cubenses II (La Habana) 2: 9. 1987.

Material examinado: (HUEFS 155055).

Subulispora longirostrata Nawawi \& Kuthub., Mycotaxon 30: 459.1987.

Material examinado: (HUEFS 155068).

Subulispora rectilineata Tubaki, Trans. Mycol. Soc. Japan 12(1): 21. 1971.

Material examinado: (HUEFS 155070).

\section{Agradecimentos}

Os autores agradecem aos Drs. Gregorio Delgado, Roland Kirschner, Uwe Braun e Xiu-Guo Zhang pela contribuição no envio de literatura. F.R. Barbosa agradece ao Conselho Nacional de Desenvolvimento Científico e Tecnológico (CNPq) pela bolsa concedida, D.A.C Almeida agradece à Coordenação de Aperfeiçoamento de Pessoal de Nível Superior (CAPES) pela bolsa concedida e L.F.P. Gusmão agradece ao CNPq (Proc. 474589/2008-0) pelo apoio financeiro.

\section{Referências Bibliográficas}

Alexopoulos, C.J.; Mims, C.W. \& Blackwell, M. 1996. Introductory Mycology. 4 ed. New York, John Wiley \& Sons.

Ando, K. \& Tubaki, K. 1984. Some undescribed hyphomycetes in the rain drops from intact leaf-surface. Transactions of the Mycological Society of Japan 25: 21-37.
Ando, K. 1992. A study of terrestrial aquatic hyphomycetes. Transactions of the Mycological Society of Japan 33: 415-425.

Barber, P.A. \& Keane P.J. 2007. A novel method of illustrating microfungi. Fungal Diversity 27: 1-10.

Barbosa, F.F. \& Gusmão, L.F.P. 2005. Two Speiropsis species (Anamorfic Fungi-Hyphomycetes) from Bahia State, Brazil. Acta Botanica Brazilica 19(3): 515-518.

Barbosa, F.R.; Gusmão, L.F.P.; Castañeda-Ruiz, R.F.; Marques, M.F.O. \& Maia, L.C. 2007. Conidial fungi from the semi-arid Caatinga biome of Brazil. New species Deightoniella rugosa \& Diplocladiella cornitumida with new records for the neotropics. Mycotaxon 102: 39-49.

Barbosa, F.R.; Gusmão, L.F.P. \& Barbosa, F.F. 2008. Fungos anamórficos (Hyphomycetes) no Semi-árido do Estado da Bahia, Brasil. Acta Botanica Brasilica 22(1): 29-36.

Barbosa, F.R.; Maia, L.C. \& Gusmão, L.F.P. 2009a. Fungos conidiais associados ao folhedo de Clusia melchiorii Gleason e C. nemorosa G. Mey. (Clusiaceae) em fragmento de Mata Atlântica, Bahia, Brasil. Acta Botanica Brasilica 23(1): 79-84.

Barbosa, F.R.; Maia, L.C. \& Gusmão, L.F.P. 2009b. Novos registros de Hyphomycetes decompositores para o Estado da Bahia, Brasil. Acta Botanica Brasilica 23(2): 323-329.

Bärlocher, F. 2009. Reproduction and dispersal in aquatic hyphomycetes. Mycoscience 50: 3-8.

Bärlocher, F. \& Kendrick, B. 1975. Leaf-Conditioning by Microorganisms. Oecologia 20: 359-362.

Belliveau, M.J.R. \& Bärlocher, F. 2005. Molecular evidence confirms multiple origins of aquatic hyphomycetes. Mycological Research 109(12): 1407-1417.

Benke, A.C.; Hall, C.A.S.; Hawkins, C.P.; Lowe-Mcconnell, R.H.; Stanford, J.A.; Suberkropp, K. \& Ward, J.V. 1988. Bioenergetic Considerations in the Analysis of Stream Ecosystems. Journal of the North American Benthological Society 7(4): 480-502.

Benner, R. Newell, S.Y.; Maccubbin, A.E. \& Hodson R.E. 1984. Relative Contributions of Bacteria and Fungi to Rates of Degradation of Lignocellulosic Detritus in Salt-Marsh Sediments. Applied and Environmental Microbiology 48(1) 36-40.

Boling, R.H.; Goodman, E.D.; Zimmer, J.O.; Cummins, K.W.; Petersen, R.C.; Van Sickle, J.A. \& Reice, S.R. 1975. Toward a model of detritus processing in a woodland stream. Ecology 56: 141-151

Bhat, D.J. \& Kendrick, B. 1993. Twenty-five new conidial fungi from the Western Ghats and the Andaman Islands (India). Mycotaxon 49: 19-90.

Castañeda-Ruiz, R.F. 2005. Metodologia en el estudio de los hongos anamorfos. In: V congresso Latino Americano de Micologia. In Anais do V congresso Latino Americano de Micologia. Brasília. 182-183.

Castañeda-Ruiz, R.F. 1988. Fungi Cubenses III. Havana, Instituto de Investigaciones Fundamentales em Agricultura Tropical "Alejandro de Humboldt".

Castañeda-Ruiz, R.F.; Abarca, G.H.; Arias, R.M.; Saikawa, M.; Minter, D.W. \& Stadler, M. 2007a. Anamorphic fungi from submerged plant material: Phaeomonilia pleiomorpha, P. corticola and Cacumisporium pleuroconidiophorum. Mycotaxon 100: 327-336.

Castañeda-Ruiz, R.F.; Guarro, J. \& Cano, J. 1996. Notes on conidial fungi. $\mathrm{X}$. A new species of Ceratosporella and some new combinations. Mycotaxon 60: 275-281.

Castañeda-Ruiz, R.F.; Gusmão, L.F.P.; Guarro, J.; Stchigel, A.M.; Stadler, M. \& Saikawa, M. 2007b. Two new anamorphic fungi from Brazil: Cacumisporium tropicale and Acrodictys irregularis. Mycotaxon 102: 91-99.

Castañeda-Ruiz, R.F.; Gusmão, L.F.P.; Heredia-Abarca, G. \& Saikawa, M. 2006. Some Hyphomycetes from Brazil. Two new species of Brachydesmiella, two new combinations for Repetophragma, and new records. Mycotaxon 95: 261-270.

Castañeda-Ruiz, R.F. \& Kendrick, W.B. 1990. Conidial fungi from Cuba I. University of Waterloo. Biological Series 33.

Castañeda-Ruiz, R.F. \& Kendrick, W.B. 1991. Ninety-nine conidial Fungi from Cuba and three from Canada. University of Waterloo, Biology Series 35.

Chan, S.Y.; Goh, T.K. \& Hyde, K.D. 2000. Ingoldian fungi in Lam Tsuen River and Tai Po Kau Forest Stream, Hong Kong. Pp. 109-118. In: Hyde, K.D.; Ho, W.H. \& Pointing, S.B. (Eds.). Aquatic Mycology across the Millennium. Hong Kong, Fungal Diversity Press. 
Chung, N. \& Suberkropp, K. 2009. Effects of aquatic fungi on feeding preferences and bioenergetics of Pycnopsyche gentilis (Trichoptera: Limnephilidae). Hydrobiologia 630: 257-269.

Cole, J.J.; Stephen R. Carpenter, S.R.; Ktchell, J.; Pace, M.L.; Solomon, C.T. \& Weidel, B. 2010. Strong evidence for terrestrial support of zooplankton in small lakes based on stable isotopes of carbon, nitrogen, and hydrogen. Proceedings of the National Academy of Sciences 99(12): 8101-81015

Cooper, J.A. 2005. New Zealand hyphomycete fungi: additional records, new species, and notes on interesting collections. New Zealand Journal of Botany 43: 323-349.

Cruz, A.C.R. \& Gusmão, L.F.P. 2009a. Fungos conidiais na Caatinga: espécies associadas ao folhedo. Acta Botanica Brasilica 23(4): 99-1012.

Cruz, A.C.R. \& Gusmão, L.F.P. 2009b. Fungos conidiais na Caatinga: espécies lignícolas. Acta Botanica Brasilica 23(4): 1133-1144.

Cruz, A.C.R.; Gusmão, L.F.P. \& Castañeda-Ruiz, R.F. 2007a. Conidial fungi from the semi-arid Caatinga biome of Brazil. Subramaniomyces pulcher sp. nov. and notes on Sporidesmium circinophorum. Mycotaxon 102: $25-32$

Cruz, A.C.R.; Gusmão, L.F.P; Leão-Ferreira, S.M. \& Castañeda-Ruiz, R.F. 2007b. Conidial fungi from the semi-arid Caatinga biome of Brazil. Diplococcium verruculosum sp. nov. and Lobatopedis longistriatum $\mathrm{sp}$. nov. Mycotaxon 102: 33-38.

Cruz, A.C.R.; Gutiérrez, A.H. \& Gusmão, F.P. 2008a. O gênero Exserticlava (Fungo Anamorfo - Hyphomycetes) no Brasil. Revista Brasileira de Botânica 31(2): 357-361.

Cruz, A.C.R. Izabel, T.S.S.; Leão-Ferreira, S.M \& Gusmão, L.F.P. 2009. Conidial fungi from the semi-arid Caatinga biome of Brazil. New species and new records of Helicosporium. Mycotaxon 110: 53- 64.

Cruz, A.C.R.; Leão-Ferreira, S.M.; Barbosa, F.R. \& Gusmão, L.F.P. 2008 b. Conidial fungi from semi-arid Caatinga biome of Brazil. New and interesting Dictyochaeta species. Mycotaxon 106: 15-27.

Cruz, A.C.R.; Marques, M.F.O. \& Gusmão, L.F.P. 2007c. Fungos anamórficos (Hyphomycetes) da Chapada Diamantina: novos registros para o Estado da Bahia e Brasil. Acta Botanica Brasilica 21(4): 847-855.

Descals, E. 2005. Diagnostic characters of propagules of Ingoldian fungi. Mycological Research 109(5): 545-555.

Descals, E. \& Moralejo, E. 2001. Water and asexual reproduction in the ingoldian fungi. Botanica Complutensis 25: 13-71.

Dix, N.J. \& Webster, J. 1995. Fungal ecology. London, Chapman \& Hall.

Ellis, M.B. 1971. Dematiaceous hyphomycetes. Commonwealth Mycological Institute, Kew. England.

Farr, D.F.; Rossman, A.Y.; Palm, M.E. \& Mccray, E.B. 2009. Fungal Databases, Systematic Botany \& Mycology Laboratory, ARS, USDA. $<$ http://nt.arsgrin.gov/fungaldatabases/>. (Acesso em 07/07/2010).

Godeas, A.M. \& Arambarri, A.M. 2007. Hifomicetes lignícolas de Tierra del Fuego (Fungi Imperfecti, Hyphomycetales). Boletín de la Sociedad Argentina de Botánica 42 (1-2): 59-69.

Goh, T.K.; Ho, W.H.; Hyde, K.D. \& Tisui, K.M. 1997. Four new species of Xylomyces form submerged wood. Mycological Research 101(11): 1323-1328.

Goh, T.K. \& Hyde, K.D. 1996. Biodiversity of freshwater fungi. Journal of Industral Microbiology 17: 328-345.

Gönczöl, J. \& Révay, Á. 2003. Treehole fungal communities: aquatic, aeroaquatic and dematiaceous hyphomycetes. Fungal Diversity 12: 19-34.

Gönczöl, J.; Révay, Á. Fisher, P.J. 1990. Notes on Vargamyces aquaticus, a water borne dematiaceous hyphomycetes. Mycotaxon 39: 301-310.

Goos, R.D; Brooks, R.D. \& Lamore, B.J. 1977. An undescribed hyphomycete from wood submerged in a Rhode Island stream. Mycologia 69(2): 280-286.

Gulis, V. 2001. Are there any substrate preferences in aquatic hyphomycetes? Mycological Research 105(9): 1088-1093.

Gusmão, L.F.P. 2003. Microfungos associados a folhas em decomposição de plantas nativas de Campos Rupestres do estado da Bahia, Brasil. Tese de Doutorado. Universidade de São Paulo, São Paulo.

Gusmão, L.F.P. \& Barbosa, F.R. 2005. Hemibeltrania (anamorphic fungi - Hyphomycetes) from the state of Bahia, Brazil. Sitientibus, Série Ciências Biológicas 5: 17-19.
Gusmão, L.F.P.; Barbosa, F.R \& Cruz, A.C.R. 2005. Espécies de Curvularia (Fungos anamórficos - hyphomycetes) no semi-árido do estado da Bahia, Brasil. Sitientibus, Série Ciências Biológicas 5(1): 12-16.

Gusmão, L.F.P. \& Grandi, R.A.P. 2001. A new Neojohnstonia species from Brazil. Mycotaxon 80: 97-100.

Gusmão, L.F.P.; Leão-Ferreira, S.M.; Marques, M.F.O. \& Almeida, D.A.C. 2008. New species and records of Paliphora from the Brazilian semiarid region. Mycologia 100(2): 306-309.

Hughes, S.J. 1958. Revisiones hyphomycetum aliquot cum appendice de nominibus rejiciendis. Canadian Journal of Botany 36: 727-836.

Hyde, K.D. \& Goh, T. 1999. Fungi on submerged wood from the River Coln, England. Mycological Research 103(12): 1561-1574.

Ingold, C.T. 1966. The Tetraradiate Aquatic Fungal Spore. Mycologia 58(1): 43-56.

Ingold, C.T. 1975. An ilustrated guide to aquatic and waterbome hyphomycetes Fungi Imperfecti. with notes on their biology. Freshwater Biological Association Science Publications 30: 155-174.

Kirk, P.M. 1985. New or interesting Microfungi XIV. Dematiaceous hyphomycetes from Mt Kenia. Mycotaxon 23: 305-352.

Kirk, P.M. 1992. New or interesting microfungi XVI. Hyphomycetes from the British Isles. Mycotaxon 43: 231-236.

Kodsueb, R.; Mckenzie, E.H.C.; Lumyong, S. \& Hyde, K.D. 2008. Diversity of saprobic fungi on Magnoliaceae. Fungal Diversity 30: 37-53.

Kohlmeyer, J. \& Volkmann-Kohlmeyer, B. 1998. A new marine Xylomyces on Rhizophora from the Caribbean and Hawaii. Fungal Diversity 1: 159-164.

Leão-Ferreira, S.M. \& Gusmão, L.F.P. 2010. Conidial fungi from the semi-arid Caatinga biome of Brazil. New species of Endophragmiella, Spegazzinia and new records for Brazil, south America and Neotropica. Mycotaxon 111: 1-10.

Marques, M.F.O.; Barbosa, F.R.; Gusmão, L.F.P.; Castañeda-Ruiz, R.F. \& Maia, L.C. 2007. Conidial fungi from the semi-arid Caatinga biome of Brazil. Cubasina microspora sp. nov., a note on C. albofusca, and some new records for South America. Mycotaxon 102: 17-23.

Marques, M.F.O.; Gusmão, L.F.P. \& Maia, L.C. 2008. Espécies de Vermiculariopsiella (Hyphomycetes) associadas a substratos vegetais em fragmento de Mata Atlântica, Serra da Jibóia, Estado da Bahia, Brasil. Revista Brasileira de Botânica 31(4): 659-665.

Mascarenhas, J.F.; Ledru, P.; Souza, S.L.; Conceição Filho, V.M.; Melo, L.F.A.; Lorenzo, C.L. \& Milési, J.P. 1998. Geologia e recursos minerais do Grupo Jacobina e da parte sul do Greenstone Belt de Mundo Novo. Série Arquivos Abertos. Companhia Baiana de Pesquisa Mineral, Salvador.

Matsushima, T. 1975. Icones Microfungorum a Matsushima Lectorum. Kobe, Published by the author.

Matsushima, T. 1993. Matsushima Mycological Memoirs n. 7. Published by the author. Kobe.

Mathuria, C. \& Chauvet, E. 2002. Breakdown of leaf litter in a neotropical stream. Journal of the North American Benthological Society 21(3): 384-396.

Medeiros, A.O.; Pascoal, C. \& Graça, M.A.S. 2009. Diversity and activity of aquatic fungi under low oxygen conditions. Freshwater Biology 54: 142-149.

Mena-Portales, J. \& Mercado-Sierra, A.M. 1987. Alguns hifomicetes de las provincias Ciudad de La Habana y La Habana, Cuba. Ecología y Sistemática, Academia de Ciencia de Cuba 17: 1-16.

Mercado-Sierra, A. \& Castañeda-Ruiz, R.F. 1987. Nuevos o raros hifomicetes de Cuba. I. Especies de Cacumisporium, Guedea, Rhinocladium, y Veronaea. Acta Botanica Cubana 50: 1-7.

Mercado-Sierra, A.; Holubová-Jechová, V. \& Mena-Portales, J. 1997. Hifomicetos dematiáceos de Cuba, Enteroblásticos. Torino, Museo Regionale di Scienze Naturali, Monografie XIII.

Milesi, J.P.; Ledru, P.; Marcoux, E.; Mougeot, R.; Johan, V.; Lerouge, C.; Sabate, P.; Bailly, L.; Respaut, J.P. \& Skipwith, P. 2002. The Jacobina Paleoproterozoic gold-bearing conglomerates, Bahia, Brazil: a "hydrothermal shear-reservoir" model. Ore Geology Reviews 19: 95-136.

Neves, M.L.C. 2005. Caracterização da vegetação de um trecho de Mata Atlântica de Encosta na Serra da Jibóia, Bahia. Dissertação de Mestrado. Universidade Estadual de Feira de Santana, Feira de Santana. 
Révay, Á. \& Gönczöl, J. 2007. Hyalocamposporium, a new genus of hyphomycetes from submerged wood. Fungal Diversity 25: 175-185.

Schindler, M.H. \& Gessner, M.O. 2009. Functional leaf traits and biodiversity effects on litter decomposition in a stream. Ecology 90(6): 1641-1649.

Schoenlein-Cruzius, I.H. \& Grandi, R.A.P. 2003. The diversity of aquatic hyphomycetes in South America. Brazilian Journal of Microbiology 34: 183-193.

Schoenlein-Cruzius, I.H.; Moreira, C.G. \& Bicudo, D.C. 2009. Aquatic Hyphomycetes in the Parque Estadual das Fontes do Ipiranga - PEFI, São Paulo, Brazil. Revista Brasileira de Botânica 32(3): 411-426.

Schubert, K. \& Braun, U. 2005. Taxonomic revision of the genus Cladosporium s.l. 4. Species reallocated to Asperisporium, Dischloridium, Fusicladium, Passalora, Pseudoasperisporium and Stenella. Fungal Diversity 20: 187-208.

Seifert, K.A. \& Gams, W. 1985. Dischloridium roseum. Mycotaxon 24: 459-461.

Shearer, C.A. \& Lane, L.C. 1983. Comparison of three techniques for the study of aquatic hyphomycete communities. Mycologia 75: 498-508.

Shearer, C.A.; Langsam, D.M. \& Longcore, J.E. 2004. Fungi in Freshwater Habitats. Pp. 513-532. In: Mueller, G.M.; Bills, G.F. \& Foster, M.S. (Eds.). Biodiversity of Fungi: Inventory and monitoring methods. San Diego, Academic Press.

Sivanesan, A. \& Alcorn, J.L. 2002. Australiasca queenslandica gen. et sp. nov. (Chaetosphaeriaceae: Ascomycota) and its anamorph Dischloridium camelliae sp. nov. from Australia. Australian Systematic Botany 15: 741-747.

Suberkropp, K.; Arsuffi, T.L. \& Anderson, J.P. 1983. Comparison of Degradative Ability, Enzymatic Activity, and Palatability of Aquatic
Hyphomycetes Grown on Leaf Litter. Applied and Environmental Microbiology. 46(1): 237-244.

Torzilli, A.P. 1982. Polysaccharidase production and cell wall degradation by several salt marsh fungi. Mycologia 74(2): 297-302.

Tsui, C.K.M. \& Berbee, M.L. 2006. Phylogenetic relationships and convergence of helicosporous fungi inferred from ribosomal DNA sequences. Molecular Phylogenetics and Evolution 39: 587- 597.

Tsui, C.K.M.; Goh, T.K.; Hyde, D.K. \& Hodgkiss, I.J. 2001. New species or records of Cacumisporium, Helicosporium, Monotosporella and Bahusutrabeeja on submerged wood in Hong Kong streams. Mycologia 93(2): 389-397.

Tsui, C.K.M.; Hyde, K.D. \& Fukushima, K. 2003. Fungi on submerged wood in the Koito River, Japan. Mycoscience 44: 55-59.

Wallace, J. B.; Eggert, S. L.; Meyer, J. L. \& Webster, J. R. 1997. Multiple Trophic Levels of a Forest Stream Linked to Terrestrial Lit ter Inputs. Science 277: 102-104.

Webster, J. \& Descals, E. 1981. Morphology, Distribution, and Ecology of Conidial Fungi in Freshwater Habitats. Pp. 295-355. In: Cole, G.T. \& Kendrick, B. (Orgs.). Biology of Conidial Fungi. New York, Academic Press.

Webster, J.R. \& Meyer, J.L. 1997. Organic Matter Budgets for Streams: A Synthesis. Journal of the North American Benthological Society 16(1): 141-161.

Wu, W. \& Zhuang, W. 2005. Sporidesmium, Endophragmiella and related genera from China. Fungal Diversity Research Series 15. Hong Kong, Fungal Diversity Press.

Yurchenko, E.O. 2001. On some wood-inhabiting dematiaceous hyphomycetes with remarkable conidia in Belarus. Mycena 1(2): 32-54.

Zare-Maivan, H. \& Ghaderian, M. 1993. Freshwater leaf-inhabiting hyphomycetes of an Iranian river. Mycologia 85: 355-357. 